Case report

\title{
Resolution of cor pulmonale after medical management in a patient with cblC-type methylmalonic aciduria and homocystinuria: a case report
}

\author{
Laurie Profitlich $^{1 *}$, Brian Kirmse ${ }^{2}$, Melissa P Wasserstein ${ }^{2}$, \\ George Diaz ${ }^{2}$ and Shubhika Srivastava ${ }^{1}$
}

Addresses: ${ }^{1}$ Department of Pediatrics, Division of Pediatric Cardiology, Mount Sinai School of Medicine, 1 Gustave L. Levy Place, Box 1201, New York, New York 10029, USA

${ }^{2}$ Department of Genetics and Genomic Sciences, Program for Inherited Metabolic Diseases, Mount Sinai School of Medicine, 1 Gustave L. Levy Place, New York, New York 10029, USA

Email: LP* - laurie.profitlich@mssm.edu; BK - brian.kirmse@mssm.edu; MPW - melissa.wasserstein@mssm.edu; GD - george.diaz@mssm.edu; SS - shubhika.srivastava@mssm.edu

* Corresponding author

Received: 8 January 2009 Accepted: 19 June 2009 Published: 30 July 2009

Cases Journal 2009, 2:8603 doi: 10.4076/1757-1626-2-8603

This article is available from: http://casesjournal.com/casesjournal/article/view/8603

(c) 2009 Profitlich et al.; licensee Cases Network Ltd.

This is an Open Access article distributed under the terms of the Creative Commons Attribution License (http://creativecommons.org/licenses/by/3.0), which permits unrestricted use, distribution, and reproduction in any medium, provided the original work is properly cited.

\begin{abstract}
We describe a 3-year-old Hispanic male with cblC-type methylmalonic aciduria and homocystinuria who presented to the emergency department with progressive tachypnea, vomiting, and edema secondary to pulmonary embolism and cor pulmonale. With aggressive medical management, there was complete resolution of right heart failure and pulmonary hypertension after 3 months. Pulmonary embolism is rare in the pediatric population. Children with cblC-type methylmalonic aciduria and homocystinuria may be at increased risk for thrombus formation and pulmonary embolism due to chronic hyperhomocystinemia, a risk factor for thrombus formation in the adult population. Aspirin therapy may be indicated in children with inborn errors of metabolism that predispose to hyperhomocystinemia.
\end{abstract}

\section{Introduction}

CblC-type methylmalonic aciduria and homocystinuria (cblC) is an inborn error of vitamin $B_{12}$ (cobalamin) metabolism. The disease is attributable to mutations in the gene MMACHC located on chromosome 1 [1]. Abnormalities in MMACHC's product result in deficient intracellular conversion of cobalamin into its two functional forms: adenosylcobalamin (AdoCbl) and methylcobalamin (MeCbl) [1]. AdoCbl normally acts as a cofactor for the mutase-dependant conversion of methylmalonyl-CoA into
succinyl-CoA in organic acid metabolism and MeCbl is the cofactor for methionine synthase in the re-methylation of homocysteine to methionine. Therefore, the molecular defect in cblC results in the accumulation of methylmalonic acid and homocysteine (Figure 1).

The clinical manifestations of cblC are heterogeneous, but typically include neurologic, developmental, ophthalmologic and hematologic abnormalities. Although patients with classic homocystinuria (cystathione beta-synthase 


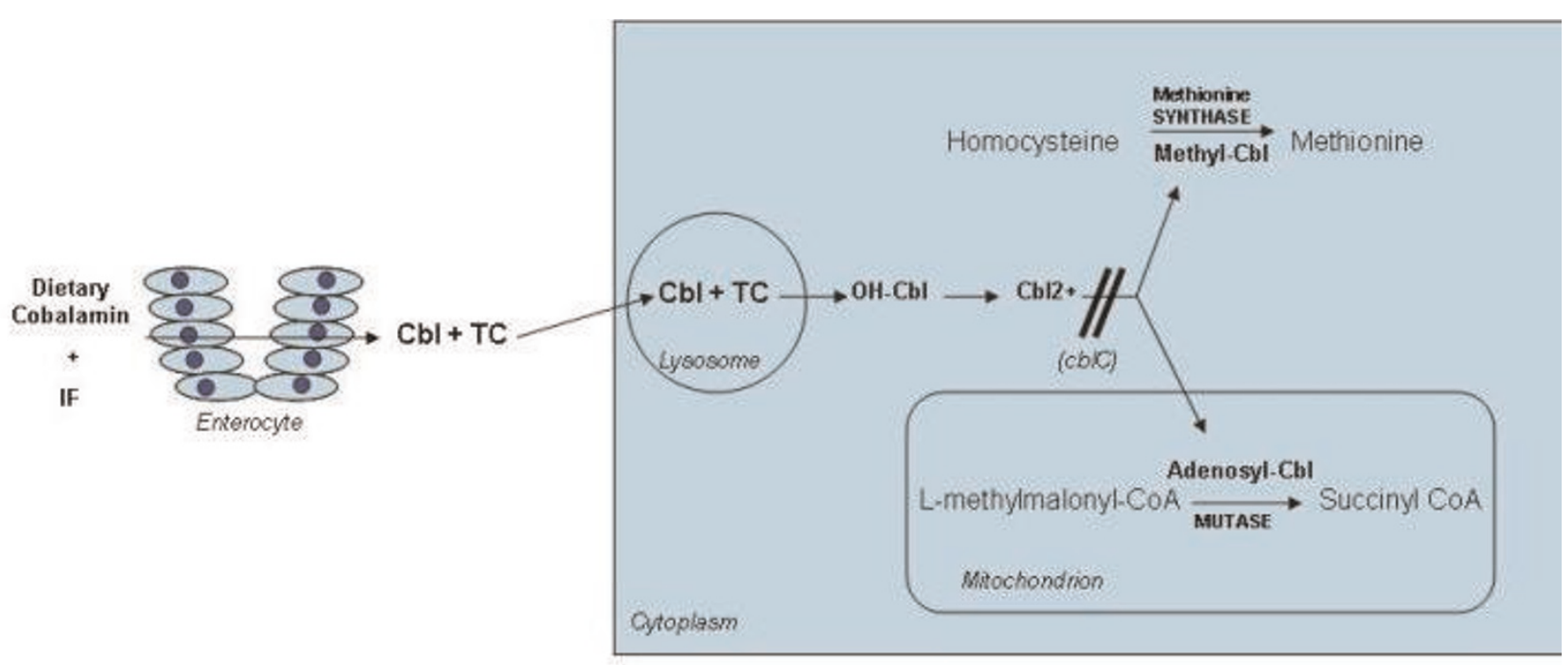

\section{$\mathrm{IF}=$ Intrinsic factor, $\mathrm{TC}=$ Transcobalamin, $\mathrm{OH}=\mathrm{Hydroxy}$}

Figure I. Schematic illustrating how the molecular defect in cblC results in the accumulation of methylmalonic acid and homocysteine.

deficiency) who present with isolated hyperhomocystinemia are at high risk for thromboembolic events, no thromboembolic events were reported in the largest case series of 50 patients with cblC $[2,3]$. There is only one case report describing an infant diagnosed post-mortem with cblC and cor pulmonale due to thromboembolism [4]. Successful medical management of cor pulmonale in this patient population has not been previously described.

\section{Case presentation}

We describe a full-term Hispanic, male infant transferred to our neonatal intensive care unit for management of hypotonia and an abnormal urinary methylmalonic acid level. The serum homocysteine level was elevated (279 micromol/L; normal range $<15 \mathrm{micromol} / \mathrm{L}$ ), suggesting a disorder of cobalamin metabolism. The diagnosis of cobalamin $\mathrm{C}$ type methylmalonic aciduria and homocystinuria (cblC) was later confirmed through molecular testing. While in the NICU, a heart murmur was detected and an echocardiogram showed a bicommissural pulmonary valve without stenosis and normal biventricular function. He was discharged from the NICU on medical management including dietary protein restriction and hydroxycobalamin injections. He did not return for cardiac follow up during the first three years of life.
At three years of age, he presented to our emergency department with a one-week history of progressive respiratory distress, vomiting and facial and lower extremity swelling. The remainder of the medical history was noncontributory. The family reported compliance with betaine therapy, but hydroxycobalamin injections were not being given.

In the emergency department, the vital signs were pulse 130 beats/min, blood pressure $100 / 70 \mathrm{mmHg}$, respiratory rate 40 breaths/min and $\mathrm{O}_{2}$ saturation $86 \%$ while breathing room air. His $\mathrm{O}_{2}$ saturation improved to $99 \%$ with $5 \mathrm{~L}$ of oxygen by facemask. He appeared to be a small (14 kg, $25^{\text {th }}$ percentile), sick child in moderate respiratory distress with significant perioral cyanosis. He had periorbital and pretibial edema. His lungs had decreased air entry at the bases, but no rales were audible. Cardiac examination revealed a hyperdynamic precordium, regular rhythm, normal S1 and a loud, single S2 with no murmurs. His liver edge was palpable $8 \mathrm{~cm}$ below the right costal margin and the spleen tip was palpable just below the left costal margin. His distal extremities were cool with delayed capillary refill of 3-4 seconds and pulses were 1+ bilaterally.

A chest radiograph showed cardiomegaly, a right lower lobe infiltrate and a small right pleural effusion. 
Laboratory investigations were significant for $\mathrm{Na} 134$, $\mathrm{CO}_{2}$ 14, BUN 42, creatinine 0.7, ALT 128, AST 247, LDH 818, d-dimer 4.34. His ABG was pH 7.33, $\mathrm{pCO}_{2} 33, \mathrm{HCO}_{3}$ 17 and base deficit of -7.6 . His total serum homocysteine level was $181 \mathrm{micromol} / \mathrm{L}$ (normal $<15 \mathrm{micromol} / \mathrm{L}$ ). The methylmalonic acid level in his urine was $90 \mathrm{micromol} / \mathrm{mol}$ $\mathrm{Cr}$ (normal range $<2 \mathrm{micromol} / \mathrm{mol} \mathrm{Cr}$ ). CBC showed mild macrocytic anemia (hematocrit 31.2\%, MCV 110.6 fL).

A 12-lead EKG showed sinus tachycardia, right axis deviation and right ventricular hypertrophy. An echocardiogram showed severe pulmonary hypertension with severe right ventricular dilation (Figure 2A), severe tricuspid regurgitation, moderate pulmonary regurgitation, mild mitral regurgitation and mildly depressed left ventricular function with a shortening fraction of $24 \%$. The right ventricular pressure was estimated to be greater than one-half of the systemic blood pressure by the tricuspid regurgitant jet (Figure 3 ). The pulmonary artery diastolic pressure was $35 \mathrm{mmHg}$ above the right ventricular end-diastolic pressure based on the pulmonary regurgitant jet (Figure 4). Additionally, there was a small pericardial effusion.

The diagnosis of cor pulmonale secondary to pulmonary embolism was suspected. In the PICU, milrinone (0.5 $\mathrm{mcg} / \mathrm{kg} / \mathrm{min}$ ), a heparin infusion (10 units $/ \mathrm{kg} / \mathrm{hr}$ ) and furosemide ( $1 \mathrm{mg} / \mathrm{kg}$ IV every 8 hours) were initiated. Hydroxycobalamin ( $1 \mathrm{mg}$ IM daily) was reinstituted and betaine therapy was optimized at a dose of 12 grams/day (850 mg/kg/day). Folic acid and pyridoxine were supplemented. A lung perfusion scan showed a large perfusion

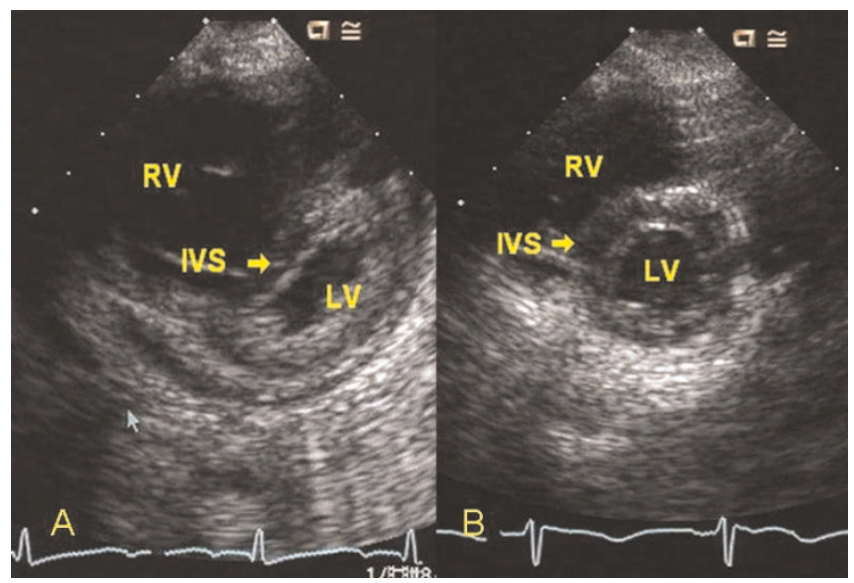

Figure 2. Parasternal short axis $2 \mathrm{D}$ echocardiographic images. (A) At the time of admission there was severe right ventricular (RV) dilation and evidence of RV hypertension with marked interventricular septal (IVS) flattening in systole. (B) At 2.5 month follow-up, the RV size is normal and there is resolution of RV hypertension with no IVS flattening in systole.

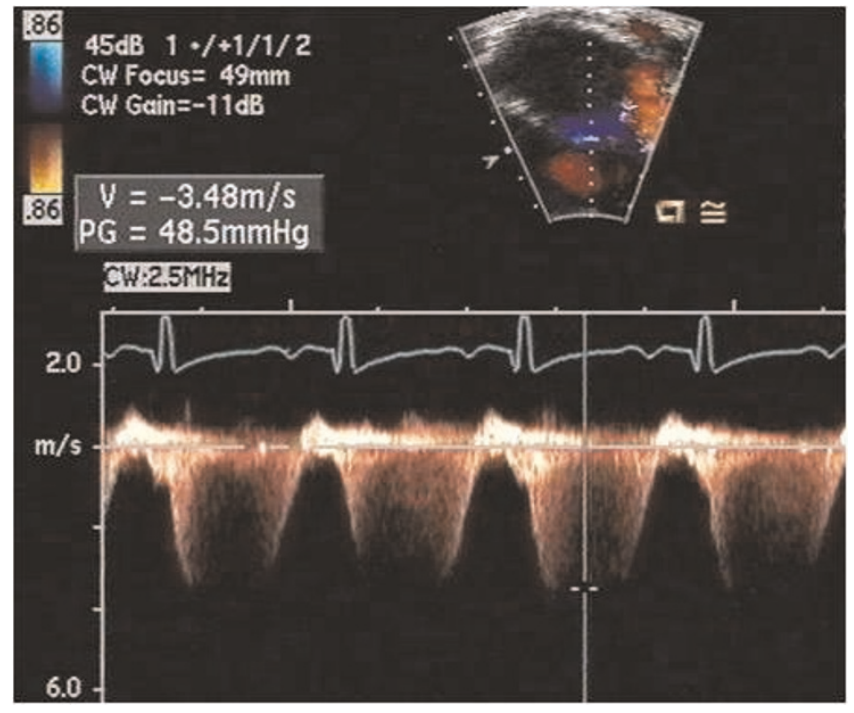

Figure 3. Spectral Doppler interrogation of severe tricuspid regurgitation showing a maximal instantaneous gradient of $48 \mathrm{mmHg}$. The right ventricular systolic pressure is estimated at $48 \mathrm{mmHg}$ plus the right atrial pressure (more than one-half systemic pressure with systolic BP of $100 \mathrm{mmHg}$ ) using the modified Bernoulli equation.

defect in the right middle lobe, but the result was indeterminate for pulmonary embolism due to poor patient cooperation. Ultrasound of the distal inferior vena cava as well as bilateral iliac, common femoral,

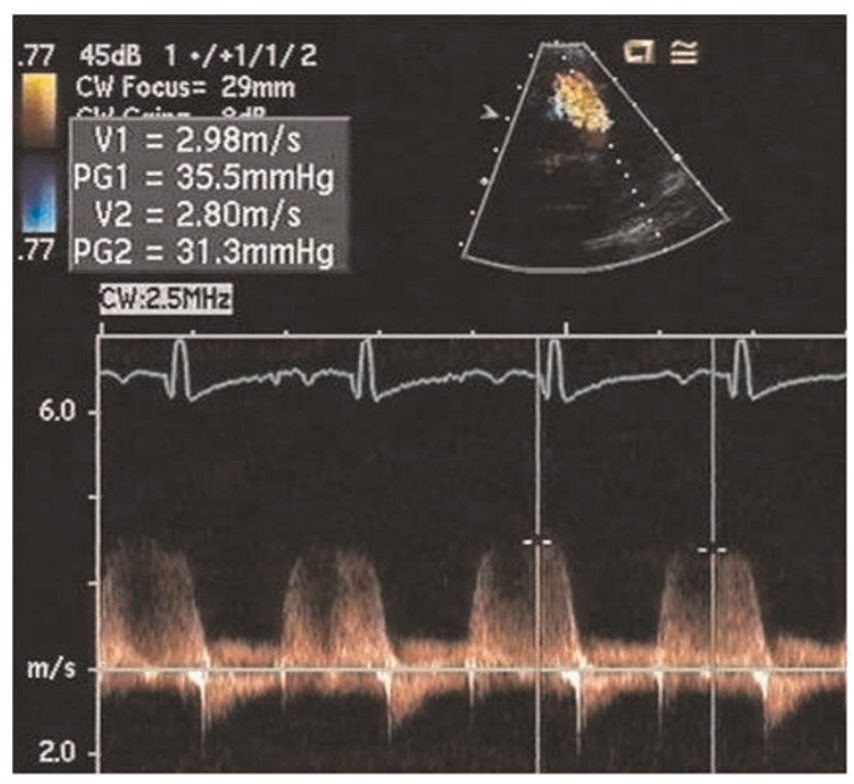

Figure 4. Spectral Doppler interrogation of the pulmonary regurgitant jet showing the pulmonary artery diastolic pressure to be elevated at $35 \mathrm{mmHg}$ above the right ventricular end diastolic pressure. 
superficial femoral and popliteal veins showed no evidence of deep vein thrombosis.

The patient tolerated the transition onto oral furosemide and digoxin. Sildenafil was initiated and his dose was increased to $5 \mathrm{mg}(0.3 \mathrm{mg} / \mathrm{kg} /$ dose $)$ PO every 8 hours. He was kept on low-molecular weight heparin every 12 hours for anticoagulation. Prior to discharge, an echocardiogram showed no significant improvement in his pulmonary hypertension, right heart function or degree of tricuspid and pulmonary regurgitation.

Since discharge, he has had no recurrence of cardiovascular symptoms. A repeat echocardiogram 2.5 months after discharge showed complete resolution of pulmonary hypertension with normal right ventricular size and function (Figure 2B), trivial tricuspid, mitral and pulmonary regurgitation and qualitatively normal left ventricular systolic function. His furosemide and digoxin were discontinued at that time. Sildenafil was continued for an additional 4 months and was weaned without recurrence of pulmonary hypertension. He remains on aspirin for anticoagulation and is followed regularly by our Program for Inherited Metabolic Diseases where his average total homocysteine level has been $67 \mathrm{micromol} / \mathrm{L}$.

\section{Discussion}

Deep vein thrombosis and pulmonary emboli are uncommon in pediatric patients [5]. A prospective Canadian registry of venous thromboembolic events (VTE) in children between the ages of 1 and 18 years showed the incidence of pulmonary embolism to be $5.3 / 10,000$ hospital admissions or $0.07 / 10,000$ Canadian children [6]. A prospective two-year registry of (VTE) in Dutch children less than 18 years showed the incidence of VTE to be $0.14 / 10,000$ children [7]. Both studies found that more than $95 \%$ of children with DVT and PE had at least one associated condition predisposing them to thromboembolism [6,7]. Patients with cblC may be predisposed to thromboembolism because of the elevated homocysteine levels associated with their disease. There are no studies reporting on the relationship between hyperhomocysteinemia and thromboembolism in pediatric patients. However, mild hyperhomocystinemia is an established independent risk factor for myocardial infarction and stroke in adults, events which are often triggered by thromboembolism $[8,9]$.

This is the first report of complete resolution of pulmonary hypertension and right heart failure after aggressive medical management in a patient with cblC. Since hyperhomocysteinemia has been shown to be an independent risk factor for thromboembolism in the adult population [10], aspirin prophylaxis may be indicated to minimize the risk of thromboembolism in pediatric patients with a predisposition for hyperhomocysteinemia. This case also underscores the importance of aggressively searching for treatable underlying conditions that predispose to thromboembolic disease, such as the inborn errors of homocysteine metabolism.

\section{Abbreviations}

ABG, arterial blood gas; AdoCbl, adenosylcobalamin; ALT, alanine transaminase; AST, aspartate aminotransferase; BUN, blood urea nitrogen; $\mathrm{CBC}$, complete blood count; cblC, Cobalamin $\mathrm{C}$ type methylmalonic aciduria and homocystinuria; $\mathrm{EKG}$, electrocardiogram; $\mathrm{HCO}_{3}$, bicarbonate; IM, intramuscular; LDH, lactate dehydrogenase; MeCbl, methylcobalamin; MCV, mean corpuscular volume; NICU, neonatal intensive care unit; $\mathrm{PE}$, pulmonary embolism; PICU, pediatric intensive care unit; VTE, venous thromboembolic events.

\section{Consent}

Written informed consent was obtained from the patient's mother for publication of this case report and accompanying images. A copy of the written consent is available for review by the Editor-in-Chief of this journal.

\section{Competing interests}

The authors declare that they have no competing interests.

\section{Authors' contributions}

LP collected the patient data and was a major contributor in writing this manuscript. BK analyzed the patient data regarding the metabolic disease and was a major contributor in writing this manuscript. LP and BK contributed equally to this work. MPW provided expertise regarding the metabolic disease and was a reviewer of this manuscript. GD provided expertise regarding the metabolic disease management and was a reviewer of this manuscript. SS was instrumental in the diagnosis and management of this patient and was a major contributor in manuscript preparation.

\section{References}

I. Lerner-Ellis JP, Tirone JC, Pawelek PD, Doré C, Atkinson JL, Watkins D, Morel CF, Fujiwara TM, Moras E, Hosack AR, Dunbar GV, Antonicka H, Forgetta V, Dobson CM, Leclerc D, Gravel RA, Shoubridge EA, Coulton JW, Lepage P, Rommens JM, Morgan K, Rosenblatt DS: Identifcation of the gene responsible for methylmalonic aciduria and homocystinuria, cblC type. Nat Genet 2006, 38:93-100.

2. Rosenblatt DS, Aspler AL, Shevell MI, Pletcher BA, Fenton WA, Seashore MR: Clinical heterogeneity and prognosis in combined methylmalonic aciduria and homocystinuria (cblC). J Inherit Metab Dis 1997, 20:528-538.

3. Mudd SH, Skovby F, Levy HL, Pettigrew KD, Wilcken B, Pyeritz RE, Andria G: The natural history of homocystinuria due to cystathionine b-synthase deficiency. Am J Hum Genet 1985, 37: I.

4. Brandstetter Y, Weinhouse E, Splaingard ML, Tang TT: Cor pulmonale as a complication of methylmalonic acidemia and homocystinuria (cbl-C type). Am J Med Genet 1990, 36:|67-|7|. 
5. Van Ommen $\mathrm{CH}$, Peters M: Acute pulmonary embolism in childhood. Thromb Res 2006, I 18:13-25.

6. Andrew M, David M, Adams M, Ali K, Anderson R, Barnard D, Bernstein M, Brisson L, Cairney B, DeSai D: Venous thromboembolic complications (VTE) in children: First analyses of the canadian registry of VTE. Blood 1994, 83:|25I-I 257

7. Van Ommen $\mathrm{CH}$, Heijboer $\mathrm{H}$, Buller HR, Hirasing RA, Heijmans HS, Peters M: Venous thromboembolism in childhood: A prospective two-year registry in the Netherlands. J Pediatr 200I, 139:676-68|.

8. Stampfer MJ, Malinow MR, Willett WC, Newcomer LM, Upson B, Ullman D, Tischler PV, Hennekens $\mathrm{CH}$ : A prospective study of plasma homocyst(e)ine and risk of myocardial infarction in US physicians. JAMA 1992, 268:877-88I.

9. Bostom AG, Rosenberg IH, Silbershatz $\mathrm{H}$, Jacques PF, Selhub J, D-Agistino RB, Wilson PW, Wolf PA: Nonfasting plasma total homocysteine levels and stroke incidence in elderly persons: the Framingham Study. Ann Intern Med 1999, 13 I:352-355.

10. Ray JG: Meta-analysis of hyperhomocysteinemia as a risk factor for venous thromboembolic disease. Arch Intern Med 1998, |58:2101-2106.

\section{Do you have a case to share?}

Submit your case report today

- Rapid peer review

- Fast publication

- PubMed indexing

- Inclusion in Cases Database

Any patient, any case, can teach us something

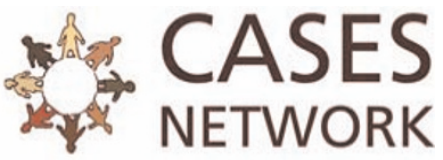

www.casesnetwork.com 